\title{
Patient similarity analytics for explainable clinical risk prediction
}

\author{
Hao Sen Andrew Fang ${ }^{1 *}$, Ngiap Chuan Tan ${ }^{1,2}$, Wei Ying Tan ${ }^{3}$, Ronald Wihal Oei ${ }^{3}$, Mong Li Lee ${ }^{3,4}$ and \\ Wynne Hsu 3,4
}

\begin{abstract}
Background: Clinical risk prediction models (CRPMs) use patient characteristics to estimate the probability of having or developing a particular disease and/or outcome. While CRPMs are gaining in popularity, they have yet to be widely adopted in clinical practice. The lack of explainability and interpretability has limited their utility. Explainability is the extent of which a model's prediction process can be described. Interpretability is the degree to which a user can understand the predictions made by a model.

Methods: The study aimed to demonstrate utility of patient similarity analytics in developing an explainable and interpretable CRPM. Data was extracted from the electronic medical records of patients with type-2 diabetes mellitus, hypertension and dyslipidaemia in a Singapore public primary care clinic. We used modified K-nearest neighbour which incorporated expert input, to develop a patient similarity model on this real-world training dataset $(n=7,041)$ and validated it on a testing dataset $(n=3,018)$. The results were compared using logistic regression, random forest (RF) and support vector machine (SVM) models from the same dataset. The patient similarity model was then implemented in a prototype system to demonstrate the identification, explainability and interpretability of similar patients and the prediction process.
\end{abstract}

Results: The patient similarity model $(A \cup R O C=0.718)$ was comparable to the logistic regression (AUROC $=0.695)$, RF $(A U R O C=0.764)$ and SVM models (AUROC $=0.766)$. We packaged the patient similarity model in a prototype web application. A proof of concept demonstrated how the application provided both quantitative and qualitative information, in the form of patient narratives. This information was used to better inform and influence clinical decisionmaking, such as getting a patient to agree to start insulin therapy.

Conclusions: Patient similarity analytics is a feasible approach to develop an explainable and interpretable CRPM. While the approach is generalizable, it can be used to develop locally relevant information, based on the database it searches. Ultimately, such an approach can generate a more informative CRPMs which can be deployed as part of clinical decision support tools to better facilitate shared decision-making in clinical practice.

Keywords: Patient similarity, Prediction models, Explainable artificial intelligence, Interpretable, Clinical decision support tool

${ }^{*}$ Correspondence: andrew.fang.h.s@singhealth.com.sg

1 SingHealth Polyclinics, SingHealth, 167, Jalan Bukit Merah, Connection

One, Tower 5, \#15-10, Singapore, P.O. 150167, Singapore

Full list of author information is available at the end of the article

\section{Introduction}

Clinical risk prediction models (CRPM) are designed to assist healthcare professionals in making better clinical decisions [1]. In general, CRPMs use patient characteristics to estimate the probability about having (or developing) a particular disease (or outcome) [2]. As healthcare 
knowledge continues to expand and outstrip human cognitive capacity, CRPM have gained popularity as they offer a scalable way to consolidate growing volumes of data and information complexity to support clinical decision-making [3]. Such CRPMs range from predicting hospital readmissions, to various types of cancers, and more recently COVID-19 [4-7].

Despite their proliferation, CRPMs have yet to be adopted in clinical practice on a larger scale $[8,9]$. While concerns regarding rigour in development and validation of CRPMs are being addressed by established guidelines, attention is shifting toward improving their explainability and interpretability [9-19]. Explainability is defined as the extent of which a model's prediction process can be described, while interpretability is defined as the degree to which a user can understand the predictions made by a model [20-22].

Recently, patient similarity analytics has become a popular technique for CRPM development [23]. The underlying concept is to identify similar patients to a patient of interest, and use them as a clinically meaningful subgroup to derive more precise prognostic information [24], and has also been shown to improve prediction accuracy $[25,26]$. One advantage of this technique is that it is able to display the similar patients that it uses to make the predictions. This increases the transparency in the prediction process, thus improving model explainability. With the similar patients, case-based narrative can thus be crafted around the predictions to enhance their interpretability.

\section{Methods}

\section{Study aim}

This study aims to demonstrate the deployment of patient similarity analytics to develop an explainable and interpretable CRPM using an electronic medical records derived dataset of patients with type-2 diabetes mellitus (D), hypertension $(\mathrm{H})$ and dyslipidaemia $(\mathrm{L})$ and their DHL-related complications in primary care.

\section{Data description}

This study was conducted using a real-world dataset consisting of de-identified electronic medical records of patients who visited a polyclinic in south-eastern Singapore. This polyclinic manages about 450 to 500 patient attendances daily during office hours and serves about 350,000 multi-ethnic Asians living in the district. About one-third of patients who attend the polyclinic are aged 65 and above. For the purpose for this study, patients who visited for any of the DHL conditions during the period of April 1, 2014 to March 31, 2015 were included in the dataset. Their demographic characteristics, disease history, laboratory test results and prescribed medications data were extracted over a 10-year period from April 1, 2009 to March 31, 2019. Ethics board approval was obtained before the conduct of this study (SingHealth Centralized Institutional Review Board Reference Number: 2019/2604).

\section{Data definitions}

The first visit of each patient during the period of April 1, 2014 to March 31, 2015 was denoted as the base visit. This was the index visit used to provide a cross-sectional representation of each patient's disease status, including years with disease, medications, and complications. The look-back period (April 1, 2009 to March 31, 2014) was used to obtain the DHL disease history, while the lookforward period (April 1, 2014 to March 31, 2019) was used to obtain data on DHL complication onset.

Patients' onset of any one or combination of DHL conditions was their earliest visit with a pre-defined set of International Classification of Disease 9th or 10th revision (ICD) codes, or relevant medications (Table 2) in the look-back period. Patients with type- 2 diabetes mellitus (D) were defined by ICD codes 250.90, 250.40, 250.80, E11.9, E11.21, E11.22, E14.31, E14.73 and E11.40, or if they were on insulin or other oral anti-diabetic medications. Patients with essential hypertension $(\mathrm{H})$ were defined by ICD codes 401.1, 796.2, I10, or if they were being treated with any one or more anti-hypertensive medications. Patients with dyslipidemia (L) were defined by ICD codes $272.0, \mathrm{E} 78.5$, or if they were taking prescribed lipid-lowering medication(s).

Patients were deemed to have DHL-related complications if their visit history in both the look-back and lookforward periods contained predefined set of ICD codes in Table 1 . In addition to the ICD codes, patients were considered to have an eye complication if they had a diabetic referrable finding on eye examination and/or were on follow-up with an eye specialist. Patients were deemed to suffer from a foot complication if they have been flagged as high risk for foot ulcer during an examination and/or were on follow-up with a podiatrist or vascular surgeon. Patients were also deemed to have kidney complication if they had estimated glomerular filtration rate $<60 \mathrm{ml} /$

Table 1 International Classification of Diseases 10 codes for eye, foot, kidney and macrovascular complications

\begin{tabular}{ll}
\hline Complication & ICD codes \\
\hline Eye & E1431, 3620 \\
Foot & E1140, E1473, 1739, 4439 \\
Kidney & E1122, 25,040, N183, N184, N185, 5859, 585 \\
Macrovascular & I249, I259, 4149, I500, 4280, G459, 164, 4349
\end{tabular}

ICD: International Classification of Diseases 
Table 2 List of variables (and their description) included in computing degree of similarity

\begin{tabular}{|c|c|c|}
\hline No & Variables & Description \\
\hline \multicolumn{3}{|c|}{ Demographic } \\
\hline 1 & Age & Age at base visit date \\
\hline \multicolumn{3}{|c|}{ Duration of disease (years) } \\
\hline 2 & Duration of diabetes & Duration of diabetes at base visit date \\
\hline 3 & Duration of hypertension & Duration of hypertension at base visit date \\
\hline 4 & Duration of hyperlipidemia & Duration of hyperlipidemia at base visit date \\
\hline \multicolumn{3}{|c|}{ Biomarkers } \\
\hline 5 & Body mass index & Body mass index at base visit \\
\hline 6 & $\mathrm{HbA1} \mathrm{c}^{\mathrm{a}}$ level (\%) & Hemoglobin A1c level at base visit date \\
\hline 7 & Systolic BP ${ }^{b}(\mathrm{mmHg})$ & Systolic blood pressure at base visit date \\
\hline 8 & Diastolic BPb (mmHg) & Diastolic blood pressure at base visit date \\
\hline 9 & $\mathrm{LDL}^{\mathrm{C}}$ level $(\mathrm{mmol} / \mathrm{L})$ & Low-density lipoprotein level at base visit date \\
\hline 10 & $\mathrm{HDL}^{\mathrm{d}}$ level (mmol/L) & High-density lipoprotein level at base visit date \\
\hline 11 & $\mathrm{TG}^{\mathrm{e}}$ level (mmol/L) & Triglyceride level at base visit date \\
\hline \multicolumn{3}{|c|}{ Anti-diabetic medications: daily dose } \\
\hline 12 & Metformin & Total daily dose of each anti-diabetic medication at base visit \\
\hline 13 & Glipizide & \\
\hline 14 & Gliclazide & \\
\hline 15 & Tolbutamide & \\
\hline 16 & Acarbose & \\
\hline 17 & Sitagliptin & \\
\hline 18 & Linagliptin & \\
\hline 19 & Dapagliflozin & \\
\hline 20 & Empagliflozin & \\
\hline 21 & Rapid-acting insulin & \\
\hline 22 & Isophane insulin & \\
\hline 23 & Insulin glargine & \\
\hline 24 & Insulin detemir & \\
\hline 25 & Pre-mixed insulin & \\
\hline \multicolumn{3}{|c|}{ Anti-hypertensive medications: daily dose } \\
\hline 26 & Candesartan & $\begin{array}{l}\text { Total daily dose of each anti-hypertensive medication at base } \\
\text { visit }\end{array}$ \\
\hline 27 & Captopril & \\
\hline 28 & Enalapril & \\
\hline 29 & Lisinopril & \\
\hline 30 & Losartan & \\
\hline 31 & Perindopril & \\
\hline 32 & Telmisartan & \\
\hline 33 & Valsartan & \\
\hline 34 & Atenolol & \\
\hline 35 & Bisoprolol & \\
\hline 36 & Propranolol & \\
\hline 37 & Amlodipine & \\
\hline 38 & Nifedipine & \\
\hline 39 & Hydrochlorothiazide & \\
\hline 40 & Indapamide & \\
\hline 41 & Spironolactone & \\
\hline 42 & Hydralazine & \\
\hline 43 & Methyldopa & \\
\hline 44 & Amiloride & \\
\hline
\end{tabular}


Table 2 (continued)

\begin{tabular}{|c|c|c|}
\hline No & Variables & Description \\
\hline \multicolumn{3}{|c|}{ Lipid-lowering medications: daily dose } \\
\hline 45 & Lovastatin & $\begin{array}{l}\text { Total daily dose of each lipid-lowering medication at base } \\
\text { visit }\end{array}$ \\
\hline 46 & Pravastatin & \\
\hline 47 & Simvastatin & \\
\hline 48 & Atorvastatin & \\
\hline 49 & Rosuvastatin & \\
\hline 50 & Fenofibrate & \\
\hline 51 & Gemfibrozil & \\
\hline 52 & Ezetimibe & \\
\hline 53 & Cholestyramine & \\
\hline \multicolumn{3}{|c|}{ Anti-diabetic medication class (count) } \\
\hline 54 & Biguanides & Count of number of medications in each class at base visit ${ }^{f}$ \\
\hline 55 & Sulphonylureas & \\
\hline 56 & Alpha-glucosidase inhibitors & \\
\hline 57 & Dipeptidyl peptidase 4 inhibitors & \\
\hline 58 & Sodium-glucose co-transporter 2 inhibitors & \\
\hline 59 & Insulin & \\
\hline \multicolumn{3}{|c|}{ Anti-hypertensive medication class (count) } \\
\hline 60 & $\begin{array}{l}\text { Angiotensin-converting enzyme inhibitors } \\
\text { and Angiotensin II receptor blockers }\end{array}$ & Count of number of medications in each class at base visit ${ }^{f}$ \\
\hline 61 & Beta blockers & \\
\hline 62 & Calcium channel blockers & \\
\hline 63 & Diuretics & \\
\hline 64 & Other anti-hypertensive classes & \\
\hline \multicolumn{3}{|c|}{ Anti-hypertensive medication class (count) } \\
\hline 65 & Statins & Count of number of medications in each class at base visit ${ }^{f}$ \\
\hline 66 & Other lipid-lowering medications & \\
\hline \multicolumn{3}{|c|}{ Medication purpose (count) } \\
\hline 67 & Anti-diabetic medications & $\begin{array}{l}\text { Count of number of medications for each condition at base } \\
\text { visit }\end{array}$ \\
\hline 68 & Anti-hypertensive medications & \\
\hline 69 & Lipid-lowering medications & \\
\hline
\end{tabular}

a HbA1c: Hemoglobin A1c

${ }^{b}$ BP: Blood pressure

c LDL: Low-density lipoprotein

d HDL: High-density lipoprotein

e TG: Triglyceride

${ }^{f}$ For these variables, the count is either 0 or 1

$\min / 1.73 \mathrm{~m}^{2}$ (based on CKD-EPI [Chronic Kidney Disease Epidemiology Collaboration] equation); and macrovascular complication if they had been prescribed the following antiplatelet medications: aspirin, clopidogrel, dipyridamole or ticagrelor. [27].

\section{Data preprocessing}

Patients who developed complications before their base visit date were excluded in this study. In this way, the study population included patients with pre-existing conditions who were at risk of developing complications only after the date of their base visit (i.e. in the subsequent 5-years).

We included only laboratory test and medication that are related to DHL conditions. These were determined a priori by clinicians managing patients with DHL, and are based on clinical practice guidelines. Additional variables, namely medication class and number of medications taken for each purpose, were derived from the individual medication data. The final list of variables in 
the dataset are found in Table 2. All the variables were continuous variables.

Missing data was handled by data imputation. For nonmedication variables, normal values were imputed except for age and body mass index which we used mean values. Table 3 shows the normal values imputed for the missing data. For the medications, a zero value was imputed for medications the patient was not taking.

\section{Patient similarity model development}

This study aimed to demonstrate that patient similarity can be used to develop an effective model for risk prediction. As such, we computed and aggregated the risk of $\mathrm{K}$ similar patients where $\mathrm{K}$ was determined using a grid-search. Min-max scaling was applied to each of

Table 3 Normal values imputed for the missing data

\begin{tabular}{lc}
\hline Variable & Imputed value \\
\hline Age (years) & $63.2^{*}$ \\
Body mass index $\left(\mathrm{kg} / \mathrm{m}^{2}\right)$ & $25.2^{*}$ \\
Systolic blood pressure $(\mathrm{mmHg})$ & $129.8^{*}$ \\
Diastolic blood pressure $(\mathrm{mmHg})$ & $70.6^{*}$ \\
$\mathrm{HbA1c}^{\mathrm{a}}(\mathrm{mmol} / \mathrm{L})$ & 6.0 \\
$\mathrm{LDL}^{\mathrm{b}}(\mathrm{mmol} / \mathrm{L})$ & 3.0 \\
$\mathrm{HDL}^{\mathrm{c}}(\mathrm{mmol} / \mathrm{L})$ & 1.0 \\
$\mathrm{TG}^{\mathrm{d}}(\mathrm{mmol} / \mathrm{L})$ & 1.7
\end{tabular}

a HbA1c: Hemoglobin A1c

${ }^{b}$ LDL: Low-density lipoprotein

c HDL: High-density lipoprotein

${ }^{d}$ TG: Triglyceride

* Mean value imputed the discrete variables. Additionally, expert input was also incorporated into the model, by obtaining weightage based on importance of each of the variables from consensus among a team of three clinicians. The weights were on a scale of 1 to 10 (1-least important, 10-most important). The expert consensus derived weights used in the model are shown in Table 4. The reason we elected to use a manual approach to deriving the weight was firstly to demonstrate how expert inputs can be incorporated into this approach of model development, and secondly to keep the model simple and easily explainable in how it derives the outputs (i.e. less numbers with many decimal points).

The distance metric used in K-nearest neighbour (KNN) is euclidean distance. Euclidean distance metric is a widely used distance measure for similarity search. Smaller distance implies higher degree of similarity. In this study, we present each patient in a vector of $\mathrm{m}$-dimensional feature space. Accordingly, patient $\mathrm{A}$ is represented as $\mathrm{A}=\left(f_{a_{1}}, f_{a_{2}}, \ldots f_{a_{m}}\right)$ and patient $\mathrm{B}$ as $\mathrm{B}=\left(f_{b_{1}}, f_{b_{2}}, \ldots f_{b_{m}}\right)$.

Mathematically, formula for the patient similarity model which uses a weighted Euclidean distance is expressed as follows:

$$
\operatorname{dist}(A, B)=\sqrt{\sum_{i=1}^{m}\left(\left(f_{a_{i}} * w_{i}\right)-\left(f_{b_{i}} * w_{i}\right)\right)^{2}}
$$

where $f_{a_{i}}$ and $f_{b_{i}}$ are the normalized $\mathrm{i}^{\text {th }}$ feature of patient $\mathrm{A}$ and patient $\mathrm{B} ; \mathrm{m}$ is total number of features; $w_{i}$ denotes the feature importance weights derived from expert consensus in Table 3. To ensure that no one feature dominates the distance function, the variables were

Table 4 Variable importance weights derived from expert consensus

\begin{tabular}{|c|c|}
\hline Variable & $\begin{array}{l}\text { Importance weight (1-least } \\
\text { important, to } 10 \text {-most } \\
\text { important) }\end{array}$ \\
\hline Age & 5 \\
\hline Number of years with condition (Diabetes, Hypertension, Hyperlipidemia) & 10 \\
\hline Body mass index & 2 \\
\hline $\mathrm{HbA} 1 \mathrm{c}^{\mathrm{a}}$ & 5 \\
\hline Blood pressure values (Systolic and diastolic) & 2.5 \\
\hline Cholesterol biomarkers $\left(\mathrm{LDL}^{\mathrm{b}}, \mathrm{HDL}^{\mathrm{c}}, \mathrm{TG}^{\mathrm{d}}\right)$ & 1.5 \\
\hline Individual medication daily dose & 1 \\
\hline Count of medications in each medication class & 2 \\
\hline Count of medications for each condition & 5 \\
\hline
\end{tabular}

\footnotetext{
a HbA1c: Hemoglobin A1c

b LDL: Low-density lipoprotein

c HDL: High-density lipoprotein

${ }^{\mathrm{d}}$ TG: Triglyceride
} 
Table 5 Hyperparameters used in the final patient similarity model

\begin{tabular}{ll}
\hline Hyperparameter & Value \\
\hline Nearest neighbours & 10 \\
Weights & Uniform \\
Metric & Euclidean distance \\
Search algorithm & Ball tree \\
\hline
\end{tabular}

normalized using minimum-maximum (MinMax) scaler with the formula as follows: $f_{i_{\text {scaled }}}=\frac{f_{i}-f_{i_{\text {min }}}}{f_{i_{\max }-}-f_{i_{\min }}}$ where $f_{i}$ is original value, $f_{i_{\text {scaled }}}$ is transformed value, $f_{i_{\text {min }}}$ and $f_{\text {max }}$ are the minimum and maximum values in feature $i$. The scaled data will range between values of 0 to 1 . In this formula, the Diabetes, Hypertension and Lipid status (and duration of disease) were treated as independent input variables to the model. The hyperparameters used in the patient similarity model are shown in Table 5 .

The patient similarity model was compared to other methods, namely logistic regression, random forest (RF) and support vector machines (SVM). They were compared using the area under receiver operating characteristic curve (AUROC) to evaluate their effectiveness in predicting DHL complications on the same dataset. A 7:3 train-test split was used for each model development and validation, with the same random seed for all methods.

All computations and analyses were conducted using open source software machine learning libraries and packages in Python 3.7 environment. To calculate the 95\% confidence intervals for the AUROC values, we additionally used the pROC package in R. The specific function uses 2000 bootstraps to perform the $95 \%$ confidence interval computation.

To demonstrate how the model generated its predictions and how the predictions can be made explainable and interpretable, a prototype system was developed to allow deployment of the patient similarity model on the full dataset to identify similar patients and to produce risk predictions for new patients not in the dataset. The prototype was packaged as a web application using the Flask framework. It was deployed as a standalone system (disconnected from the electronic medical record system).

\section{Results}

A total of 16,144 unique patients who visited the polyclinic for DHL between April 1, 2014 and March 31, 2015 was initially included in the dataset. 6,085 of them developed any one of the complications prior to the base visit date and were removed from the final dataset. The characteristics of the 10,059 remaining patients used in study are presented in Table 6.

Patients in the dataset had a mean age of $63.2 \pm 11.3$ years with a higher proportion of females (59.9\%). The cohort also had a bias towards the combination of Hypertension and Hyperlipidemia (41.1\%). The second most prevalent condition among the cohort of patient is Hyperlipidemia (22.1\%), followed by the Diabetes, Hypertension and Hyperlipidemia combination

Table 6 Baseline characteristics of study patients

\begin{tabular}{|c|c|c|}
\hline & $n=10,059$ & Missing, $\mathrm{n}(\%)$ \\
\hline \multicolumn{3}{|l|}{ Characteristics } \\
\hline Age (years), mean (SD) & $63.2(11.3)$ & $0(0.0)$ \\
\hline Sex, males, n (\%) & $4131(41.1)$ & $0(0.0)$ \\
\hline Race, n (\%) & & $0(0.0)$ \\
\hline Chinese & $8455(84.1)$ & \\
\hline Malay & $635(6.3)$ & \\
\hline Indian & $532(5.3)$ & \\
\hline Others & $437(4.3)$ & \\
\hline Body mass index $\left(\mathrm{kg} / \mathrm{m}^{2}\right)$, mean (SD) & $25.2(4.5)$ & $1433(14.2)$ \\
\hline Systolic BP (mmHg), mean (SD) & $129.8(17.7)$ & $60(0.6)$ \\
\hline Diastolic BP (mmHg), mean (SD) & $70.6(10.8)$ & $60(0.6)$ \\
\hline $\mathrm{HbA} 1 c^{\mathrm{a}}(\%)$ & $7.1(1.4)$ & $7712(76.6)^{*}$ \\
\hline $\mathrm{LDL}^{\mathrm{b}}(\mathrm{mmol} / \mathrm{L})$ & $3.1(0.9)$ & 2175 (21.6)\# \\
\hline $\mathrm{HDL}^{\mathrm{c}}(\mathrm{mmol} / \mathrm{L})$ & $1.5(0.4)$ & $2124(21.1) \#$ \\
\hline $\mathrm{TG}^{\mathrm{d}}(\mathrm{mmol} / \mathrm{L})$ & $1.4(0.9)$ & $2124(21.1)$ \\
\hline Diagnosis, n (\%) & & $0(0.0)$ \\
\hline Diabetes only & $150(1.5)$ & \\
\hline Hypertension only & $1501(14.9)$ & \\
\hline Hyperlipidemia only & $2223(22.1)$ & \\
\hline Diabetes \& Hypertension & $149(1.5)$ & \\
\hline Diabetes \& Hyperlipidemia & $315(3.1)$ & \\
\hline Hypertension \& Hyperipidemia & $4133(41.1)$ & \\
\hline Diabetes, Hypertension \& Hyperlipidemia & $1588(15.8)$ & \\
\hline Complications, n (\%) & & Not applicable ${ }^{\varrho}$ \\
\hline Eye, n (\%) & $1180(11.7)$ & \\
\hline Foot, n (\%) & $117(1.2)$ & \\
\hline Kidney, n (\%) & $811(8.1)$ & \\
\hline Macrovascular, n (\%) & $1119(11.1)$ & \\
\hline Any of the above, $n(\%)$ & $2590(25.7)$ & \\
\hline Look-back duration (years), mean (SD) & $4.1(1.3)$ & Not applicable ${ }^{\complement}$ \\
\hline Look-forward duration (years), mean (SD) & $4.1(1.5)$ & Not applicable ${ }^{\complement}$ \\
\hline \multicolumn{3}{|l|}{ a HbA1c: Hemoglobin A1c } \\
\hline \multicolumn{3}{|l|}{ b LDL: Low-density lipoprotein } \\
\hline \multicolumn{3}{|l|}{${ }^{c}$ HDL: High-density lipoprotein } \\
\hline \multicolumn{3}{|c|}{ d TG: Triglyceride } \\
\hline \multicolumn{3}{|c|}{$\begin{array}{l}\text { * High number of missing values as not all patients had Diabetes to require a } \\
\text { Hemoglobin A1c test }\end{array}$} \\
\hline \multicolumn{3}{|c|}{$\begin{array}{l}\text { \# Discrepancy between LDL and HDL values as some patients had extremely } \\
\text { high TG to invalidate calculated LDL }\end{array}$} \\
\hline
\end{tabular}


(15.8\%). A total of 2,509 (25.7\%) patients in this study cohort developed at least one complication within five years after the base visit, with eye complications (11.7\%) being the most common type.

With an initial $\mathrm{K}$ value of 5 , the patient similarity model achieved an AUROC of 0.688 (0.667 to 0.709) in predicting DHL complications. The grid search (sensitivity analysis) yielded the best $\mathrm{K}$ value of 10 , and the patient similarity model achieved an AUROC of 0.718 (0.697 to 0.739) (see Table 7). Compared with the other models, the patient similarity-based model was shown to be more accurate than logistic regression $(\mathrm{AUROC}=0.695)$, and slightly less accurate as the SVM $($ AUROC $=0.766)$ and RF $($ AUROC $=0.764)$ models.

With regard to the clinician assigned weights, we found that it helped to improve the model performance. When removing the variable importance weights shown in

Table 7 Comparison of patient similarity model performance with other models

\begin{tabular}{ll}
\hline Model & AUROC (95\% Cl) \\
\hline Patient similarity $(K=10)$-weighted & $0.718(0.697$ to 0.739$)$ \\
Patient similarity $(K=10)$-unweighted & $0.688(0.667$ to 0.709$)$ \\
Logistic regression & $0.695(0.672$ to 0.718$)$ \\
Random forest & $0.764(0.744$ to 0.784$)$ \\
Support vector machine (kernel = linear) & $0.766(0.746$ to 0.785$)$ \\
\hline
\end{tabular}

Table 3, the patient similarity model had a poorer performance (AUROC $=0.688$ ). While the coefficient values or Gini importances of individual medications was similarly low across all the two models, which corresponds with the expert consensus for the patient similarity model, there were differences in the way the variables were ranked between the models. The logistic regression coefficients and RF Gini importances can be found in the Additional file 1: Table 1.

Patient similarity model explainability and interpretability The patient similarity model was implemented as a web application to allow users to enter details about a new patient and to generate an estimated risk of DHL complications (see Fig. 1).

In terms of explainability, this approach is transparent in how it generates its risk predictions. The first step is to perform a multi-dimensional search across 69 variables, with importance weights applied, to find the ten most similar patients, based on Euclidean distance. The next step is to then aggregate the known outcomes of these ten patients from the database to compute the risk. For example, if four out of the ten patients had a DHL complication, the estimated risk for the new patient would be $40 \%$.

In terms of interpretability, for the same example above, the predicted risk can be understood by patients as "based on the ten most similar patients to myself, four

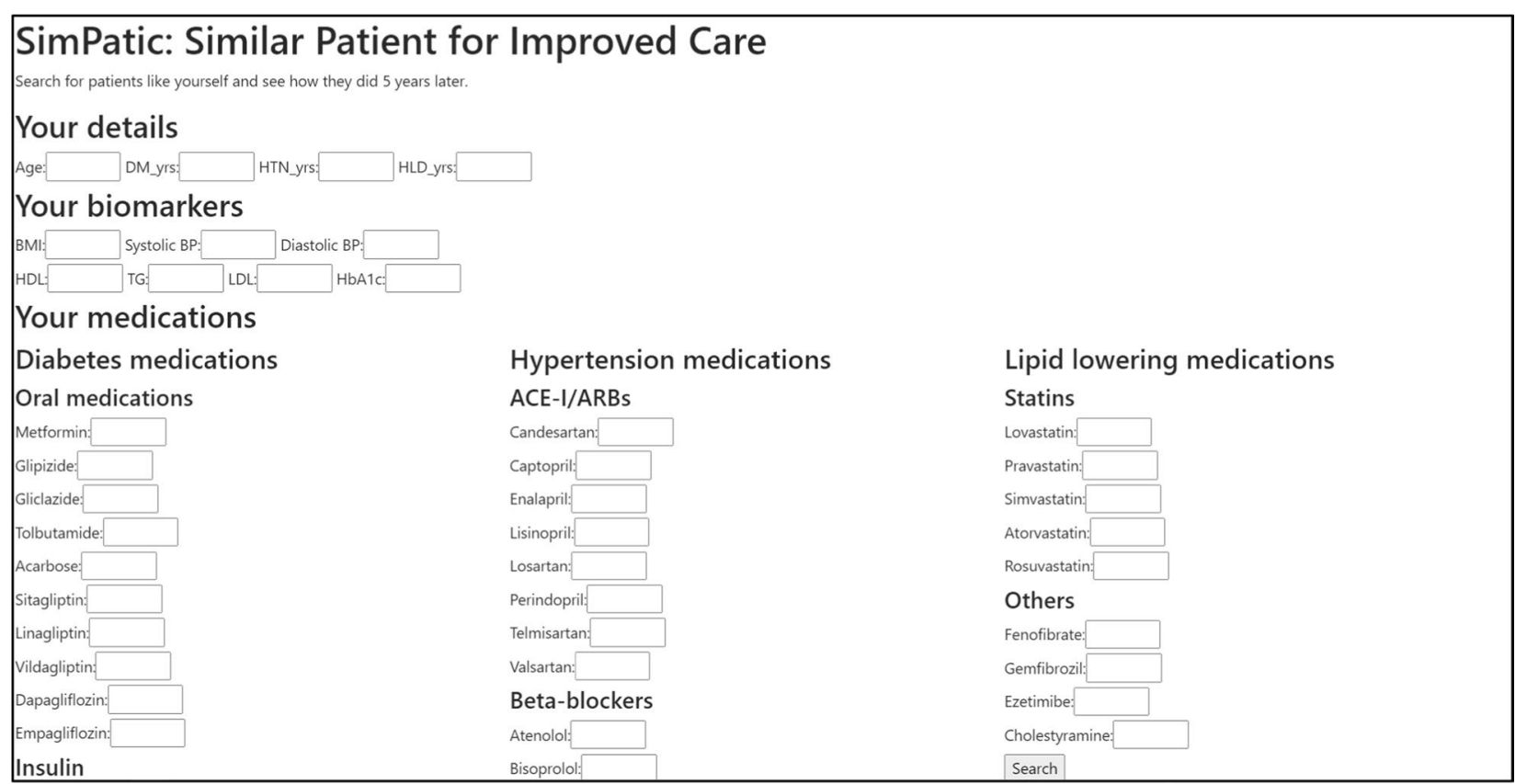

Fig. 1 The landing page (zoomed in at 175\%) of the prototype web application using the patient similarity model. Users can enter demographic, biomarker and medication inputs to identify similar patients from the database 
in ten of them had a DHL complication within the next 5 years". Furthermore, with the ability to pinpoint the ten most similar patients, healthcare providers can select a particular similar patient to view his/her longitudinal medical history over the subsequent five years. This could be used as a basis for crafting a more compelling narrative to deliver prognostic information.

\section{Proof of concept}

To illustrate how the web application can be used, we conducted mock consultation with a young patient with poorly controlled diabetes (Patient X). The attending doctor (primary user of the application) entered relevant details of Patient $\mathrm{X}$ in the web application. Patient X was 40 years old with pre-existing Diabetes, Hypertension and Hyperlipidemia for 4 years, 5 years and 5 years respectively. He had poorly controlled Diabetes with HbA1c of $10.0 \%$. He was taking metformin (total daily dose [TDD]: $2000 \mathrm{mg}$ ), and glipizide (TDD: $20 \mathrm{mg}$ ), lisinopril (TDD: $20 \mathrm{mg}$ ), amlodipine (TDD: $10 \mathrm{mg}$ ) and atorvastatin (TDD: $20 \mathrm{mg}$ ) (see Fig. 2).

The backend system would identify the top-10 most similar patients from the database of 10,059 patients and display them as a list of anonymised records (see Fig. 3). In this case, among the top-10 most similar patients to Patient X, four of them had developed a complication. This can be interpreted by Patient $\mathrm{X}$ to be "for the 10 most similar patients to myself, four had a DHL complication in the next five years." The attending doctor would leverage on such prognostic information to prompt Patient $\mathrm{X}$ to take action to optimize his/her glycemic control.

Going one step further, the system also allows the attending doctor to select a particular similar patient to generate a timeline. In this case, the attending doctor selects Patient \#10,845 who is a 59 year old with Diabetes, Hypertension and Hyperlipidemia each for 5 years. Patient \#10,845 also has poorly controlled Diabetes with HbA1c of $10.1 \%$. From the timeline, it shows Patient \#10,845 starting Insulin Glargine and later increasing the dose of the medication to eventually achieve good glycemic control and staved off all complications (see Fig. 4). Using this timeline information, the attending doctor would be able to craft a casebased narrative to recommend Patient X to start Insulin Glargine to achieve glycemic control. Conversely, the attending doctor can select a patient, who has developed a complication, to present an adverse scenario to alert Patient X.

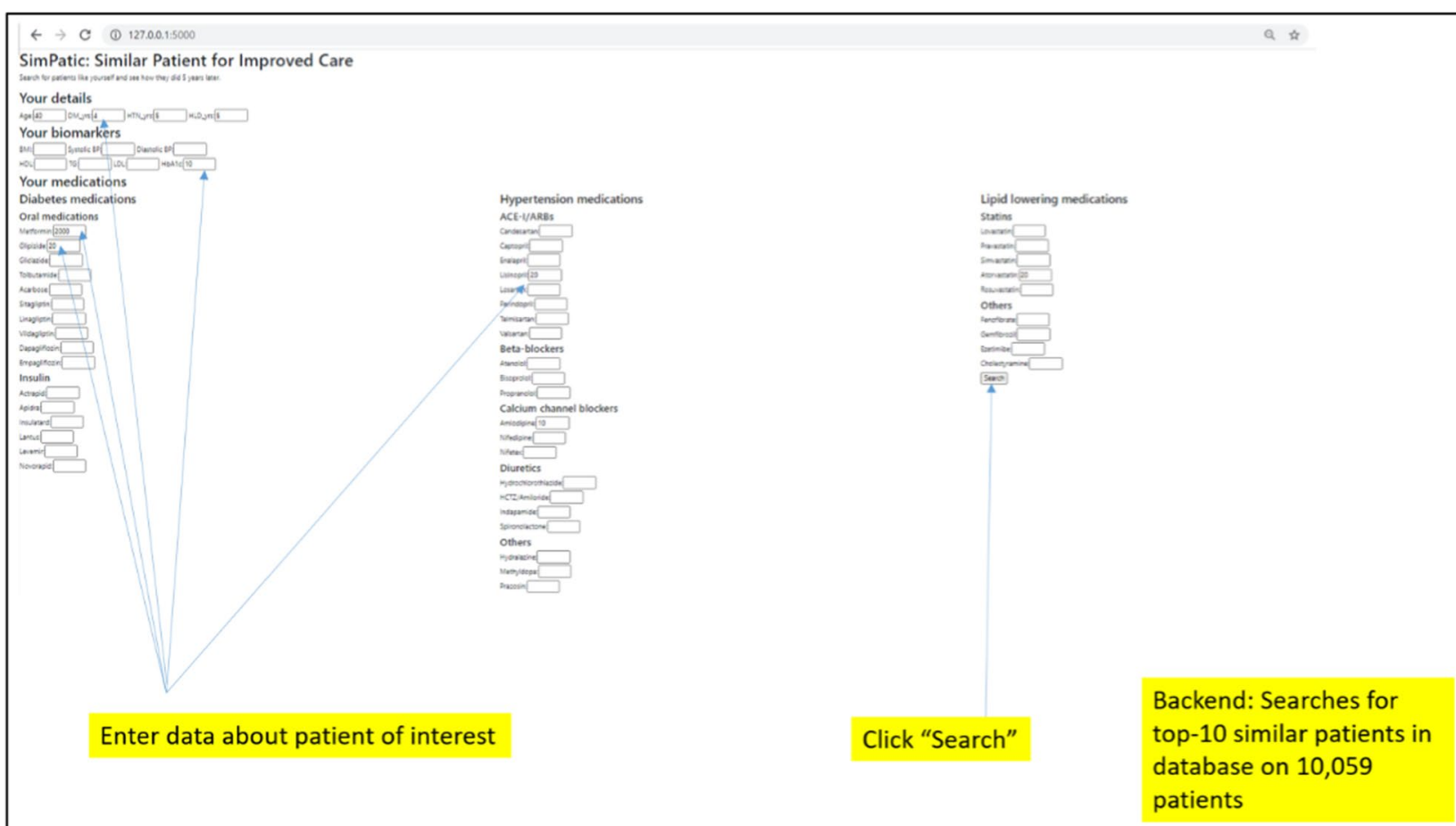

Fig. 2 Data input into the prototype web application. The attending doctor enters the details of Patient $X$ into the web application. Fields are non-mandatory. After entering the details, the attending doctor clicks the "Search" button which triggers the patient similarity model to identify the top-10 most similar patients in the database 


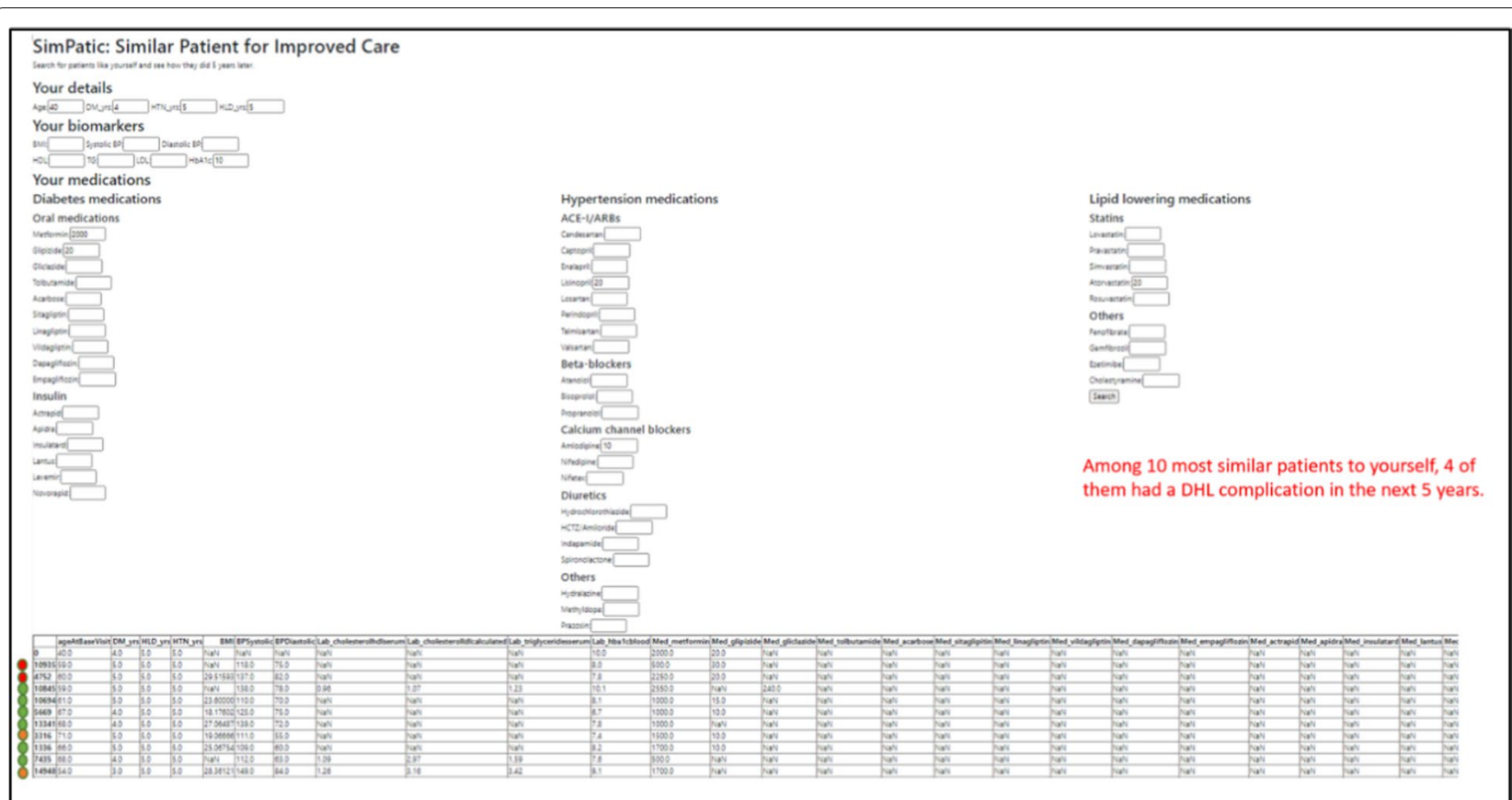

Fig. 3 An anonymized list of the top-10 most similar patients to Patient X is presented. An aggregate prognostic value is calculated based on the proportion of the top-10 patients who encountered a DHL complication. The green/orange/red indicators represent the outcomes of each patient over the subsequent 5 years from base visit. Green indicates that the patient did well (i.e. no complications). Orange indicates the patient had some complications or worsening in biomarker, while red indicates that the patient did poorly with multiple complications. In this case, four of the ten patients had either orange or red indicators

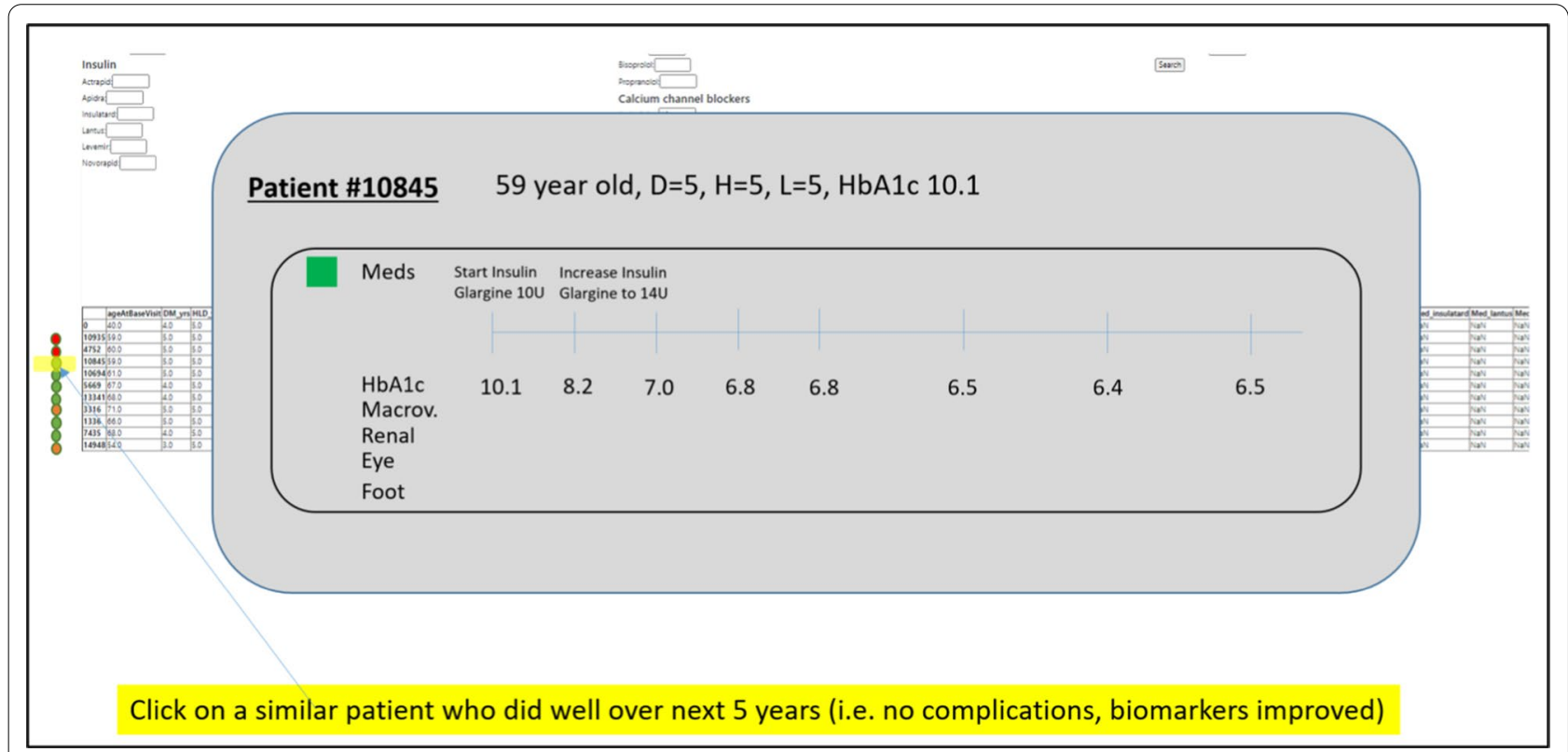

Fig. 4 Timeline of a similar patient (Patient \#10845). A particular similar patient can be selected to produce a timeline. In this case, Patient \#10845 was selected to illustrate to Patient X a patient like himself who did well, and what Patient \#10845 did to achieve the good results 


\section{Discussion}

In this study, we presented an approach of using a conventional machine learning technique, namely K-nearest neighbor, which can incorporate expert clinician knowledge to develop a patient similarity model for DHL complications prediction. For usage of the model, we proposed a two-layered presentation of information to patients. The first layer presents an aggregated risk of similar patients, while the second layer presents a narrative of a similar patient. The latter addresses a gap in conventional risk models, such as logistic regression models. Furthermore, unlike conventional risk models which provide more abstract predictions (i.e. "this is your estimated risk"), patient similarity models can couch the informational output more realistically (i.e. "this is what actually happened to $\mathrm{X}$ out of $\mathrm{Y}$ patients similar to yourself"). Given that different doctors and patients have unique preferences for the type and style of information delivery, patient similarity models will probably not replace conventional risk models, but complement them with a new variety of information that can be selectively used in the appropriate consultation context.[28].

Previous work had employed different strategies to develop explainable prediction models [9, 16-19]. Shickel et al. used a self-attention approach to highlight time steps in their model's input time series that the model believes to be most important in formulating the final mortality prediction. This was visualized in a two-dimensional grid [16]. Zhang et al. also developed an attention based prediction model and used a heatmap to present the relative importance of events over time [17]. While Rajkomar et al. explored using free text data within the dataset to enhance explainability, Lundberg et al. presented several tools like dependence plots and explanation embeddings to better explain tree-based model outputs $[18,19]$.

In spite of these developments, adopting them in clinical settings remains a challenge. Our patient similarity approach is easy to use and may be applied to various settings, diseases and patient groups. As long as there is an available database of patient records, a patient similarity CRPMs can be developed. Using this approach, they can be contextualized to the local patient characteristics and type of data variables in the database, which can then be used to develop an end-product that is locally relevant and applicable.

Complementing hard facts with patient stories have been shown to be an effective means of patient education by increasing personal relevance and reducing counterarguing [29]. Bokhour et al. showed that an education intervention using patients' success stories in controlling their hypertension resulted in more emotional engagement and reported intentions to change behavior [30].
This is further supported by Lesselroth and Monkman who have advocated embedding powerful narratives and stories in health information technology and for further research and development to evaluate its effectiveness [31]. In this way, our idea of using similar patients to craft narratives for CRPMs is an elegant way of weaving together qualitative and quantitative prognostic information to support decision-making.

While our experimental patient similarity model may not have the best performance in terms of discriminatory power, it was able to achieve an acceptable AUROC comparable to other machine learning methods such as SVM and RF [32]. With fine-tuning of other hyperparameters and ongoing research into novel similarity metrics and algorithms, patient similarity models may be able to perform even better in future [24]. For now, there is a trade-off between accuracy and explainability. In addition, unlike other CRPMs which generate a probabilistic output for a particular patient, the patient similarity model risk estimates are interpreted on the basis of "what actually happened to patients like yourself", rather than "what will happen to you". Based on this perspective, we posit that the validation of patient similarity models may not need to be as heavily scrutinized as other types of CRPMs before deployment.

We acknowledge that there are several limitations in this current patient similarity model. Firstly, it does not use all the variables, such as gender, race, diet and lifestyle, which are associated with DHL complications. The reason we did not include diet and lifestyle was because these data were not available in the datasets. While gender and race are used in some clinical risk scores, they were not included in our patient similarity model as their associations with DHL complications are comparatively weak.[33, 34] Furthermore, although we had different races in our study populations, they were mostly South Asian. This ethnic homogeneity would have lessened the value of including the race variable. If the variables were not used in the patient similarity model, they were also not used in all the other models used for comparison. Secondly, the current patient similarity model uses input data from a single time point. This would not completely be reflective of DHL which are chronic diseases. While we have included the duration of these diseases, plotting out the trajectories of these would be even more useful. Such disease progression models have been shown to be effective at predicting cardiovascular risk of diabetes and lipid disorders.[35, 36] These progression models or trajectories can be incorporated as additional input variables to improve model performance further. Thirdly, because the model building and validation included right-censored cases, it could have introduced bias. This is probably not very significant in our case since majority 
of the cases were not censored, but approaches such as inverse probability of censoring weights should be considering in datasets where larger proportion of censored cases are used.[37] Fourthly, such an approach of using K-nearest neighbour would rely on having a database of patient information to search for similar patients. This may require regular updates to the database, requiring additional effort to maintain. Furthermore, this may also encounter some data security concerns, which may be alleviated by removing personally identifiable information from the database. Lastly, the interpretability of the outputs would depend on the complexity of the cases and the size of the data. For example, in some cases this approach may not be able to find sufficient number of similar patients, by the fact that the case of interest is an outlier. In this case, the model should flag that it is not applicable. This would be similar to other models which do not perform well on outlier type of cases. However these could potentially be addressed in this approach by having an as large as possible dataset, and with as much details, to perform the search on.

Looking ahead, patient similarity analytics can be used to develop effective, explainable and interpretable CRPMs as clinical decision support and shared-decision making tools to enhance patient care. The patient similarity model will be fine-tuned and optimized with research to create optimal training hyperparameters, including search algorithms and similarity metrics. We will also explore other methods of deriving feature weights such as multivariate feature selection and Mahalanobis distance with a trainable covariance weight matrix and other machine learning model training. We will assess a to-be-developed patient similarity based tool for clinical decision support in clinical practice. For this, we plan to conduct a multi-site hybrid implementation trial to determine its impact on decision-making quality, patient and clinician satisfaction, patient health outcomes and process outcomes (such as consultation duration). Ultimately, we look forward to the tool being integrated within our electronic medical records and other IT systems, and clinical workflows.

\section{Conclusion}

In this study, we have presented patient similarity as an approach to develop an explainable and interpretable CRPM. The patient similarity model is comparable to other machine learning based models in predicting DHL-related complications. Furthermore, we introduced a prototype system to demonstrate transparency in the prediction process and the utility of the generated results to craft patient narratives. A proof of concept illustrates how this can be used in clinical practice. Adopting a patient similarity approach in developing CRPM can result in the development of more explainable and interpretable clinical decision support tools to ultimately enhance the decision-making process in clinical practice.

\begin{abstract}
Abbreviations
AUROC: Area under the receiver operating characteristic curve; BP: Blood pressure; CKD-EPI: Chronic kidney disease epidemiology collaboration; CRPM: Clinical risk prediction models; DHL: Diabetes, hypertension and Hyperlipidemia; EMR: Electronic medical records; HbA1c: Glycosylated haemoglobin; HDL: High-density lipoprotein; ICD: International classification of diseases; KNN: K-nearest neighbour; LDL: Low-density lipoprotein; RF: Random forest; SVM: Support vector machines; TDD: Total daily dose; TG: Triglyceride.
\end{abstract}

\section{Supplementary Information}

The online version contains supplementary material available at https://doi. org/10.1186/s12911-021-01566-y.

Additional file 1. Comparison of variable weights between logistic regression, random forest and those derived from expert consensus methods.

\section{Acknowledgements}

We would like to thank Ms Usha Sankari and Mr Aau Wai Keong from SingHealth Polyclinics Research Department for their support in making this work possible, and Ms Liu Hang and Ms Gao Qiao from Institute of Data Science, National University Singapore for their input in the development of the web application.

\section{Authors' contributions}

FHSA and TNC conceptualized and designed the study, with input from WYT, ORW, HW and LML. FHSA performed the data analysis. FHSA wrote the initial draft of the paper, to which the rest of the authors provided comments. All authors reviewed and approved the final manuscript.

\section{Funding}

This research is supported by the National Research Foundation, Singapore under its AI Singapore Programme (AISG Award No: AISG-GC-2019-001). Any opinions, findings and conclusions or recommendations expressed in this material are those of the authors and do not reflect the views of National Research Foundation, Singapore.

Availability of data and materials

The datasets analyzed during the current study are not publicly available as they contain information that are sensitive to the institution. They may be made available from the corresponding author on reasonable request.

\section{Declarations}

Ethics approval and consent to participate

Ethics board approval was obtained from the SingHealth Centralized Institutional Review Board (Reference Number: 2019/2604) prior to conduct of the study. The requirement of written consent was also waived by the Sing Health Centralized Institutional Review Board as it was deemed impracticable while privacy risks were mitigated through the use of de-identified data. All methods were carried out in accordance with relevant guidelines and regulations.

\section{Consent for publication}

Not applicable.

\section{Competing interests}

The authors declare no competing interests.

\section{Author details}

${ }^{1}$ Sing Health Polyclinics, SingHealth, 167, Jalan Bukit Merah, Connection One, Tower 5, \#15-10, Singapore, P.O. 150167, Singapore. ${ }^{2}$ Family Medicine 
Academic Clinical Programme, SingHealth-Duke NUS Academic Medical Centre, Singapore, Singapore. Institute of Data Science, National University of Singapore, Singapore, Singapore. ${ }^{4}$ School of Computing, National University of Singapore, Singapore, Singapore.

Received: 14 January 2021 Accepted: 22 June 2021

Published online: 01 July 2021

\section{References}

1. Wee L, van Kuijk SMJ, Dankers FJWM, Traverso A, Welch M, Dekker A. Reporting standards and critical appraisal of prediction models. In: Kubben P, Dumontier M, Dekker A, editors. Fundamentals of clinical data science [Internet]. Cham (CH): Springer; 2019 [cited 2020 Dec 7]. Available from: http://www.ncbi.nlm.nih.gov/books/NBK543529/

2. Hendriksen JMT, Geersing GJ, Moons KGM, deGroot JAH. Diagnostic and prognostic prediction models. J Thromb Haemost JTH. 2013;11 (1):129-41.

3. Shipe ME, Deppen SA, Farjah F, Grogan EL. Developing prediction models for clinical use using logistic regression: an overview. J Thorac Dis. 2019;11(Suppl 4):S574-84.

4. Mahmoudi E, Kamdar N, Kim N, Gonzales G, Singh K, Waljee AK. Use of electronic medical records in development and validation of risk prediction models of hospital readmission: systematic review. BMJ. 2020;369:m958

5. Louro J, Posso M, Hilton Boon M, Román M, Domingo L, Castells X, et al. A systematic review and quality assessment of individualised breast cancer risk prediction models. Br J Cancer. 2019;121(1):76-85.

6. Kaiser I, Pfahlberg AB, Uter W, Heppt MV, Veierød MB, Gefeller O. risk prediction models for melanoma: a systematic review on the heterogeneity in model development and validation. Int J Environ Res Public Health. 2020 Oct 28;17(21).

7. Leeuwenberg AM, Schuit E. Prediction models for COVID-19 clinical decision making. Lancet Digit Health. 2020;2(10):e496-7.

8. Dekker FW, Ramspek CL, van Diepen M. Con: most clinical risk scores are useless. Nephrol Dial Transplant Off Publ Eur Dial Transpl Assoc - Eur Ren Assoc. 2017;32(5):752-5.

9. Li R, Yin C, Yang S, Qian B, Zhang P. Marrying medical domain knowledge with deep learning on electronic health records: a deep visual analytics approach. J Med Internet Res. 2020;22(9):e20645.

10. Collins GS, Reitsma JB, Altman DG, Moons KGM. Transparent reporting of a multivariable prediction model for individual prognosis or diagnosis (TRIPOD): the TRIPOD statement. BMJ. 2015;350:97594.

11. Topol EJ. High-performance medicine: the convergence of human and artificial intelligence. Nat Med. 2019;25(1):44-56.

12. Lauritsen SM, Kristensen M, Olsen MV, Larsen MS, Lauritsen KM, Jørgensen MJ, et al. Explainable artificial intelligence model to predict acute critical illness from electronic health records. Nat Commun. 2020;11(1):3852.

13. Zihni E, Madai VI, Livne M, Galinovic I, Khalil AA, Fiebach JB, et al. Opening the black box of artificial intelligence for clinical decision support: A study predicting stroke outcome. PLOS ONE. 2020;15(4):e0231166.

14. Thorsen-Meyer HC, Nielsen AB, Nielsen AP, Kaas-Hansen BS, Toft P, Schierbeck J, et al. Dynamic and explainable machine learning prediction of mortality in patients in the intensive care unit: a retrospective study of high-frequency data in electronic patient records. Lancet Digit Health. 2020 Apr;2(4):e179-91.

15. Goldstein BA, Navar AM, Pencina MJ, loannidis JPA. Opportunities and challenges in developing risk prediction models with electronic health records data: a systematic review. J Am Med Inform Assoc JAMIA. 2017;24(1):198-208.

16. Shickel B, Loftus TJ, Adhikari L, Ozrazgat-Baslanti T, Bihorac A, Rashidi P. DeepSOFA: a continuous acuity score for critically III patients using clinically interpretable deep learning. Sci Rep. 2019;9(1):1879.

17. Zhang J, Kowsari K, Harrison JH, Lobo JM, Barnes LE. Patient2Vec: a personalized interpretable deep representation of the longitudinal electronic health record. IEEE Access. 2018;6:65333-46.

18. Rajkomar A, Oren E, Chen K, Dai AM, Hajaj N, Hardt M, et al. Scalable and accurate deep learning with electronic health records. NPJ Digit Med. 2018;1:18.
19. Lundberg SM, Erion G, Chen H, DeGrave A, Prutkin JM, Nair B, et al. From local explanations to global understanding with explainable ai for trees. Nat Mach Intell. 2020;2(1):56-67.

20. Bibal A, Lognoul M, Streel A, Frénay B. Legal requirements on explainability in machine learning. Artif Intell Law. 2020 Jul 30;

21. Watson J, Hutyra CA, Clancy SM, Chandiramani A, Bedoya A, llangovan $\mathrm{K}$, et al. Overcoming barriers to the adoption and implementation of predictive modeling and machine learning in clinical care: what can we learn from US academic medical centers? JAMIA Open. 2020;3(2):167-72.

22. Stiglic G, Kocbek P, Fijacko N, Zitnik M, Verbert K, Cilar L. Interpretability of machine learning-based prediction models in healthcare. WIREs Data Min Knowl Discov. 2020;10(5):e1379.

23. Brown S-A. Patient similarity: emerging concepts in systems and precision medicine. Front Physiol. 2016;7:561.

24. Parimbelli E, Marini S, Sacchi L, Bellazzi R. Patient similarity for precision medicine: a systematic review. J Biomed Inf. 2018;83:87-96.

25. Hassan S, Syed Z. From netflix to heart attacks: Collaborative filtering in medical datasets. IHI'10 - Proceedings of the 1st ACM International Health Informatics Symposium. 2010.128 p.

26. Wang N, Huang Y, Liu H, Fei X, Wei L, Zhao X, et al. Measurement and application of patient similarity in personalized predictive modeling based on electronic medical records. Biomed Eng OnLine. 2019;18(1):98.

27. Levey AS, Stevens LA, Schmid CH, Zhang YL, Castro AF, Feldman HI, et al. A new equation to estimate glomerular filtration rate. Ann Int Med. 2009;150(9):604-12.

28. Dyson J, Marsh C, Jackson N, Richardson D, Faisal M, Scally AJ, et al. Understanding and applying practitioner and patient views on the implementation of a novel automated Computer-Aided Risk Score (CARS) predicting the risk of death following emergency medical admission to hospital: qualitative study. BMJ Open. 2019;9(4):026591.

29. Fix GM, Houston TK, Barker AM, Wexler L, Cook N, Volkman JE, et al. A novel process for integrating patient stories into patient education interventions: incorporating lessons from theater arts. Patient Educ Couns. 2012;88(3):455-9.

30. Bokhour BG, Fix GM, Gordon HS, Long JA, DeLaughter K, Orner MB, et al. Can stories influence African-American patients' intentions to change hypertension management behaviors? A randomized control trial. Patient Educ Couns. 2016;99(9):1482-8.

31. Lesselroth B, Monkman H. Narratives and stories: novel approaches to improving patient-facing information resources and patient engagement. Stud Health Technol Inform. 2019;9(265):175-80.

32. Hosmer D, Lemeshow S. Area under the ROC curve. Appl Logist Regres. 2000;1:160-4.

33. Dagliati A, Marini S, Sacchi L, Cogni G, Teliti M, Tibollo V, et al. Machine learning methods to predict diabetes complications. J Diabetes Sci Technol. 2018;12(2):295-302.

34. Bashiri Y, Arani M, Bagheri N. Investigating factors associated with diabetes complications among Type 2 diabetic patients. J Res Med Dent Sci. 2018 May; 10:6.

35. Oh W, Kim E, Castro MR, Caraballo PJ, Kumar V, Steinbach MS, et al. Type 2 diabetes mellitus trajectories and associated risks. Big Data. $2016 \mathrm{Mar}$ 1:4(1):25-30.

36. Dayimu A, Wang C, Li J, Fan B, Ji X, Zhang T, et al. Trajectories of lipids profile and incident cardiovascular disease risk: a longitudinal cohort study. J Am Heart Assoc. 2019;8(21):013479.

37. Vock DM, Wolfson J, Bandyopadhyay S, Adomavicius G, Johnson PE, Vazquez-Benitez $\mathrm{G}$, et al. Adapting machine learning techniques to censored time-to-event health record data: A general-purpose approach using inverse probability of censoring weighting. J Biomed Inform. 2016 Jun;61:119-31.

\section{Publisher's Note}

Springer Nature remains neutral with regard to jurisdictional claims in published maps and institutional affiliations. 